\title{
Predicted Area Under the Curve Percent Extrapolation
}

National Cancer Institute

\section{Source}

National Cancer Institute. Predicted Area Under the Curve Percent Extrapolation. NCI

Thesaurus. Code C85788.

The percentage of the area under the curve (AUC) extrapolated to infinity from Tlast based on the predicted value for concentration at Tlast. 